\title{
Assessment of antimycobacterial activities of pure compounds extracted from Thai medicinal plants against clarithromycin- resistant Mycobacterium abscessus
}

\author{
Auttawit Sirichoat ${ }^{\text {Equal first author, } 1,2}$, Irin Kham-ngam ${ }^{\text {Equal first author, } 1,2}{ }^{2}$, Orawee Kaewprasert ${ }^{1,2}{ }$, Pimjai Ananta ${ }^{1,3}$, Awat \\ Wisetsai $^{4}$, Ratsami Lekphrom ${ }^{4}$, Kiatichai Faksri ${ }^{\text {Corresp. } 1,2}$

Background: Infection with Mycobacterium abscessus is usually chronic and is associated with clarithromycin resistance. Increasing drug resistance is a major public-health problem and has led to the search for new antimycobacterial agents. We evaluated the antimycobacterial activity, toxicity, and synergistic effects of several plant secondary metabolites against $M$. abscessus.

Methods: Twenty-three compounds were evaluated for antimycobacterial activity against thirty $M$. abscessus clinical isolates by broth microdilution to determine their minimum inhibitory concentration (MIC) values. Toxicity was evaluated using red and white blood cells (RBCs and WBCs). The compounds were used in combination with clarithromycin to investigate the possibility of synergistic activity.

Results: Five out of twenty-three compounds (RL008, RL009, RL011, RL012 and RL013) exhibited interesting antimycobacterial activity against $M$. abscessus, with MIC values ranging from $<1$ to $>128$ $\mu \mathrm{g} / \mathrm{mL}$. These extracts did not induce hemolytic effect on RBCs and displayed low toxicity against WBCs. The five least-toxic compounds were tested for synergism with clarithromycin against seven isolates with inducible clarithromycin resistance and seven with acquired clarithromycin resistance. The best synergistic results against these isolates were observed for RL008 and RL009 (8/14 isolates; 57\%).

Conclusions: This study demonstrated antimycobacterial and synergistic activities of pure compounds extracted from medicinal plants against clarithromycin-resistant $M$. abscessus. This synergistic action, together with clarithromycin, may be effective for treating infections and should be further studied for the development of novel antimicrobial agents. 


\section{Assessment of antimycobacterial activities of pure compounds extracted from}

\section{Thai medicinal plants against clarithromycin-resistant Mycobacterium}

3 abscessus

4

5 Auttawit Sirichoat $^{1,2 \uparrow}$, Irin Kham-ngam ${ }^{1,2 \uparrow}$, Orawee Kaewprasert ${ }^{1,2}$, Pimjai Ananta ${ }^{1,3}$, Awat

6 Wisetsai $^{4}$, Ratsami Lekphrom ${ }^{4}$ and Kiatichai Faksri ${ }^{1,2^{*}}$

$8{ }^{1}$ Department of Microbiology, Faculty of Medicine, Khon Kaen University, Khon Kaen,

9 Thailand

$10{ }^{2}$ Research and Diagnostic Center for Emerging Infectious Diseases, Khon Kaen University,

11 Khon Kaen, Thailand

$12{ }^{3}$ Clinical Laboratory Unit, Srinagarind Hospital, Faculty of Medicine, Khon Kaen University,

13 Khon Kaen, Thailand

${ }^{4}$ Natural Products Research Unit, Department of Chemistry, and Center for Innovation in

Corresponding Author: Kiatichai Faksri ${ }^{1,2^{*}}$, Department of Microbiology, Faculty of Medicine,

Khon Kaen University, Khon Kaen 40002, Thailand, Tel +6689-4373782.

E-mail address: kiatichai@kku.ac.th

I These authors contributed equally to this work.

Short title: Plant secondary metabolites against clarithromycin-resistant $M$. abscessus 
24 Abstract

25 Background: Infection with Mycobacterium abscessus is usually chronic and is associated with clarithromycin resistance. Increasing drug resistance is a major public-health problem and has

27

led to the search for new antimycobacterial agents. We evaluated the antimycobacterial activity, toxicity, and synergistic effects of several plant secondary metabolites against M. abscessus.

Methods: Twenty-three compounds were evaluated for antimycobacterial activity against thirty M. abscessus clinical isolates by broth microdilution to determine their minimum inhibitory concentration (MIC) values. Toxicity was evaluated using red and white blood cells (RBCs and WBCs). The compounds were used in combination with clarithromycin to investigate the possibility of synergistic activity.

Results: Five out of twenty-three compounds (RL008, RL009, RL011, RL012 and RL013) exhibited interesting antimycobacterial activity against $M$. abscessus, with MIC values ranging from $<1$ to $>128 \mu \mathrm{g} / \mathrm{mL}$. These extracts did not induce hemolytic effect on RBCs and displayed low toxicity against WBCs. The five least-toxic compounds were tested for synergism with clarithromycin against seven isolates with inducible clarithromycin resistance and seven with acquired clarithromycin resistance. The best synergistic results against these isolates were observed for RL008 and RL009 (8/14 isolates; 57\%).

Conclusions: This study demonstrated antimycobacterial and synergistic activities of pure compounds extracted from medicinal plants against clarithromycin-resistant M. abscessus. This synergistic action, together with clarithromycin, may be effective for treating infections and should be further studied for the development of novel antimicrobial agents.

Keywords: antimycobacterial activity, medicinal plants, plant secondary metabolites, Mycobacterium abscessus, clarithromycin resistance 


\section{Introduction}

48

49

50

51

52

53

54

55

56

57

58

Nontuberculous mycobacteria (NTM) are found in the environment (soil and water) (Velayati et al. 2014). Some species of NTM can cause life-threatening human diseases with a high mortality rate (Cassidy et al. 2009; Iroh Tam et al. 2015). Mycobacterium abscessus is a common NTM species causing chronic infection and is highly associated with drug resistance (Tung et al. 2015). Drug-resistant M. abscessus infection has become a serious health issue in many countries, including Thailand (Kham-Ngam et al. 2018). Treatment of M. abscessus infection is prolonged and one-third of cases are associated with treatment failure (Nessar et al. 2012). Although clarithromycin is a drug of choice, half of the strains present in Thailand are clarithromycin-resistant (Ananta et al. 2018). Therefore, new treatment alternatives are needed to overcome drug-resistant $M$. abscessus infection.

Plants are a source of bioactive compounds that can treat various diseases (Rios \& Recio 2005). Several research teams have reported anti-Mycobacterium tuberculosis activity of extracts from Tetradenia riparia (Baldin et al. 2018), Persea americana (Jimenez-Arellanes et al. 2013), Lophira lanceolata (Nkot et al. 2018) and Flourensia cernua (Molina-Salinas et al. 2006).

Northeastern Thailand has high plant diversity, which remains locally important as a source of traditional medicines (Kaewpiboon et al. 2012). In Thailand, extracts from Neonothopanus nambi (Kanokmedhakul et al. 2012) and Rothmannia wittii (Chaipukdee et al. 2016) showed antimycobacterial activity against $M$. tuberculosis. Atalantiaphyllines A-G, isolated from roots of Atalantia monophylla, exhibited higher aromatase inhibition than did ketoconazole, and also showed high $\alpha, \alpha$-diphenyl- $\beta$-picrylhydrazyl (DPPH) radical-scavenging activity (Pailee et al. 2020). Anthracene and anthraquinone metabolites isolated from Prismatomeris filamentosa showed antibacterial activities against Gram-positive and Gram-negative bacteria such as 
Bacillus subtilis, Bacillus cereus, Staphylococcus aureus, Escherichia coli, Pseudomonas

aeruginosa and Shigella sonnei (Wisetsai et al. 2021). The compounds derived from luminescent mushroom Neonothopanus nambi exhibited antimalarial activity against Plasmodium falciparum (Kanokmedhakul et al. 2012). However, the effect of these plant extracts against M. abscessus has not been reported.

We aimed to evaluate the antimycobacterial activities of pure compounds extracted from four medicinal plants (A. monophylla, P. filamentosa, Ageratum conyzoides and R. wittii) and from the cultured mycelium of the luminescent mushroom N. nambi. These compounds were tested against clarithromycin-susceptible and non-susceptible M. abscessus clinical isolates. The toxicity for mammalian cells and synergistic effects of selected compounds with clarithromycin were also analyzed.

(1)

\section{Materials and methods}

\section{Pure compounds extracted from local medicinal plants}

A total of 23 pure compounds, 22 isolated from four medicinal plants (A. monophylla, $P$. filamentosa, A. conyzoides and $R$. wittii) and one compound isolated from the cultured mycelium of a luminescent mushroom (N. nambi), were evaluated (Table 1 and Fig. S1). The compounds were purified using column chromatography techniques to isolate the pure secondary metabolites, as described previously (Sombatsri et al. 2018). Stock solutions were prepared by dissolving pure compounds in dimethyl sulfoxide (DMSO), which can dissolve both polar and non-polar compounds.

\section{Bacterial isolates}


94 Srinagarind Hospital, Khon Kaen University, Thailand. The colony morphology of each isolate

95

96

97

was noted. The identification of M. abscessus species was performed according to the protocol previously published (Kham-Ngam et al. 2019). All isolates were sub-cultured on LowensteinJensen (LJ) media and incubated at $37^{\circ} \mathrm{C}$ for 7 days before further analysis. Informed consent was not required for this study. Anonymized, left-over specimens were used. All specimens, including isolates and blood samples, had been obtained during routine practice. This study was approved by the Khon Kaen University Ethics Committee for Human Research (HE611496).

\section{Antibiotic susceptibility testing}

The minimum inhibitory concentration (MIC) for clarithromycin was determined according to published protocols (Kham-Ngam et al. 2019). The broth-microdilution method using a RAPMYCOI Sensititre 96-well plate (TREK Diagnostic Systems, Ohio, USA) following the manufacturer's protocol. Briefly, individual colonies of $M$. abscessus were suspended in demineralized water to obtain a density corresponding to McFarland Standard 0.5. Then, $50 \mu \mathrm{L}$ of cell suspension were transferred into a tube of cation-adjusted Mueller-Hinton broth (TREK Diagnostic Systems) with TES buffer (to optimize the conditions for antibiotic stability) to achieve a final cell concentration of approximately $5 \times 10^{5} \mathrm{CFU} / \mathrm{mL}$. One-hundred microliters of this inoculum were added to each well of a 96-well plate containing di $\square$ erent concentrations of antibiotics. The 96 -well microtiter plates were then incubated at $37^{\circ} \mathrm{C}$ for $3-14$ days under aerobic condition. Clarithromycin susceptibility was read at 3, 5, and 14 days according to CLSI guidelines (CLSI 2018). A reading at day 3 was used to test for inducible resistance according to previously described protocols (Ananta et al. 2018). Inducible resistance was inferred by changes 
116 in MIC values from "susceptible" at day 3 to "resistant" at day 14. Isolates that were resistant on

117 day 3 and thereafter were regarded as demonstrating acquired resistance. All clarithromycin-

118 susceptible and clarithromycin-resistant (both inducible and acquired resistance) M. abscessus

119 clinical isolates were used for further analysis.

120

121

\section{Antimycobacterial assay}

122

The antimycobacterial assay was carried out using a broth-microdilution method to

determine the MIC values according to the CLSI guidelines (CLSI 2018). Two-fold serial

124 dilutions of pure compounds were prepared directly in a 96-well microtiter plate. For preparation

of the M. abscessus inoculum, the same protocol as described above was used. One-hundred

microliters of this inoculum were mixed with $100 \mu \mathrm{L}$ of pure compound (to give the final

concentrations: $1,2,4,8,16,32,64$ and $128 \mu \mathrm{g} / \mathrm{mL}$ ) and were then added to each well of the 96-

well plate. Following incubation for $3-5$ days at $37^{\circ} \mathrm{C}$, MICs were visually determined as the

lowest concentration of the compound that completely inhibited the mycobacterial growth (Ananta et al. 2018).

\section{Hemolytic assay}

Hemolytic activity was determined according to protocols published previously with some modification (Lima Viana et al. 2018). Briefly, six mL of blood from a single healthy volunteer were collected and transferred into a heparin collection tube. Whole blood was centrifuged at 5,000 rpm for $5 \mathrm{~min}$ and the plasma was then discarded. Concentrated red blood cells (RBCs) were isolated and washed three times with 1\% sterile phosphate buffer saline (PBS) solution ( $\mathrm{pH} 7.4$ ) and centrifugation. Then, the RBCs were diluted in $1 \%$ PBS to a 5\% final 
139 concentration of RBC suspension for analysis $(950 \mu \mathrm{L}$ of $1 \%$ PBS and $50 \mu \mathrm{L}$ of concentrated

140 RBCs in a total volume of $1 \mathrm{~mL}$ ). Two hundred microliters of this RBC suspension were

141 transferred into the tubes containing different concentrations of pure compounds in a total

142 volume of $1 \mathrm{~mL}$ (at concentrations before adding the RBC suspension: 1, 2, 4, 8, 16, 32, 64 and

$143128 \mu \mathrm{g} / \mathrm{mL}$ in $1 \%$ PBS). Positive and negative controls were used, these being 1\% Triton X-100

144 solution (Lima Viana et al. 2018) and 1\% PBS, respectively. The final volume of each

145 experiment was $1.0 \mathrm{~mL}$. The solutions were incubated at $37^{\circ} \mathrm{C}$ for $1 \mathrm{~h}$. After incubation, the

146 suspensions were then centrifuged at $3,000 \times g$ for $2 \mathrm{~min}$. Then, $100 \mu \mathrm{L}$ of supernatant from each

147 tube were transferred into a 96-well plate for measurement of the absorbance at $540 \mathrm{~nm}$ using a

148 microplate reader (each absorbance was measured twice). In addition, RBC morphology was

149 observed under a light microscope and recorded. All tests were performed in duplicate for each

150 test compound. Hemolytic activity was calculated by the following equation:

$$
\text { Hemolysis }(\%)=[(A s-A n) /(A c-A n)] \times 100
$$

Where As refers to the absorbance of the sample, $A n$ refers to the absorbance of the

153

154

155

156

157

158

159

160

161

\section{Cell viability assay}

To assay the toxicity of each tested compound for human white blood cells, the trypanblue exclusion test was used (Strober 2015). Briefly, white blood cells (WBCs) were isolated from six mL of blood from a healthy volunteer using the Ficoll density-gradient technique (Boyum 1976). Whole blood was carefully transferred into a tube containing Ficoll solution (ratio 1:1). Then, the cells were centrifuged at $1,500 \mathrm{rpm}$ for $10 \mathrm{~min}$ at $20^{\circ} \mathrm{C}$ and the $\mathrm{WBC}$ layer 
162 was transferred into a new tube. The concentrated WBCs were washed three times with $1 \%$ PBS

163 at $1,500 \mathrm{rpm}$ for $5 \mathrm{~min}$ at $20^{\circ} \mathrm{C}$ and re-suspended in $1 \mathrm{~mL}$ of RPMI-1640 media (Gibco ${ }^{\mathrm{TM}}$, New

164 York, USA). Fifty microliters of WBC suspension were transferred into individual wells of a 96-

165 well plate and then $50 \mu \mathrm{L}$ of pure compounds at different concentrations (ranging from 1 to 128

$166 \mu \mathrm{g} / \mathrm{mL})$ were added. The $96-w e l l$ plate was incubated at $37^{\circ} \mathrm{C}$ for $1 \mathrm{~h}$. Then, $20 \mu \mathrm{L}$ of the

167 suspension was mixed with $20 \mu \mathrm{L}$ of $0.4 \%$ trypan blue solution in buffered isotonic salt solution

$168\left(0.81 \% \mathrm{NaCl}\right.$ and $\left.0.06 \% \mathrm{~K}_{2} \mathrm{HPO}_{4}\right)$ and incubated for $3 \mathrm{~min}$ at room temperature. Viable and dead

169 cells were counted under a light microscope using a hemocytometer. As a negative control, WBC

170 suspension was treated with PBS. The test was performed in duplicate for each test compounds.

171 Dead cells were calculated using the following equation:

172 Dead cells $(\%)=($ number of dead cells $/$ total number of cells $) \times 100$

174 Genome sequencing and analysis

Genomic DNA of the 30 M. abscessus clinical isolates was extracted using the cetyltrimethylammonium bromide-sodium chloride (CTAB) method (De Almeida et al. 2013) and was sent for genome sequencing (NovogeneAIT, Hong Kong) using an Illumina HiSeq platform generating $150-\mathrm{bp}$ paired-end reads. quality paired-end reads were then mapped to the M. abscessus ATCC 19977 reference genome (GenBank accession number CU458896.1) using BWA-mem (v.0.7.17) (Li 2013). For converting SAM to BAM format, sorting and indexing the bam files, SAMtools v0.1.19 algorithm was used (Li et al. 2009). GATK version 4.0.5. (McKenna et al. 2010) was used for 
185 realignment, generating coverage statistics and mapping details. Both GATK and SAMtools

186 were used for variant calling and filtering, including single-nucleotide polymorphisms (SNPs)

187 and small indels (Li et al. 2009; McKenna et al. 2010).

188 For phylogenetic analysis, a WGS-based phylogeny was analyzed using mpileup, VCF

189 and coverage files. Maximum-likelihood analysis was performed using MEGA-7 (Qasim et al.

190 2018) with the general time-reversible (GTR) and gamma model. Support for individual nodes

191 was assessed using 1000 bootstrap replicates. The phylogenetic tree was visualized using iTol

192 software (https://itol.embl.de/).

193

194 Synergism

195 Combinations of plant secondary metabolites and clarithromycin were evaluated using a 196 microdilution checkerboard method (Garcia 2010). Five concentrations of each test compound 197 (ranging from 32 to $512 \mu \mathrm{g} / \mathrm{mL}$ ) and eight concentrations of clarithromycin (Sigma-Aldrich, 198 Missouri, USA) (ranging from 32 to 4,096 $\mu \mathrm{g} / \mathrm{mL}$ ) were prepared, and M. abscessus cell 199 suspensions, prepared as for previous experiments, were used. Fifty microliters of each pure 200 compound and clarithromycin were mixed in a 96-well plate, and $100 \mu \mathrm{L}$ of inoculum were then 201 added (final concentration of each pure compound ranged from 8 to $128 \mu \mathrm{g} / \mathrm{mL}$ and of 202 clarithromycin ranged from 4 to $1,024 \mu \mathrm{g} / \mathrm{mL}$ ). The plate was incubated at $37^{\circ} \mathrm{C}$ for 7 days (for 203 isolates with acquired resistance) or 14 days (for isolates with inducible resistance). The results 204 were recorded and interpreted as the fractional inhibitory concentration index (FICI) (Doern 205 2014). The FICI value was calculated using the following equation:

206

$$
\mathrm{FICI}=[\mathrm{A}] /(\mathrm{A})+[\mathrm{B}] /(\mathrm{B})
$$



refers to MIC (B) in combination with (A), and (B) refers to MIC (B) alone. "synergy", "additive", "indifference” and "antagonism”, respectively.

\section{Data analysis}

All quantitative data are reported as means. Comparison of quantitative data among groups of the toxicity assays was performed using one-way ANOVA followed by post-hoc LSD tests. Number of isolates with colony morphotypes and the susceptibility to the compounds and clarithromycin were compared using Fisher's exact test. $P$-values $<0.05$ were considered statistically significant. All statistical analyses were performed using SPSS version 19.0 (IBM, Armonk, NY, USA).

Results

Identification and characteristics of $M$. abscessus isolates used

Thirty clinical isolates of M. abscessus on LJ solid medium were recovered. 


\section{Antimycobacterial activity of plant secondary metabolites}

The MIC values of 23 pure compounds were determined by broth microdilution against

233

234

235

236

237

238

239

240

241

242

243

244

245

246

247

248

249

250

251

252

30 clinical isolates of $M$. abscessus, including clarithromycin-susceptible and -resistant isolates.

The MIC cut-off value of $128 \mu \mathrm{g} / \mathrm{mL}$ for at least one isolate was chosen for the selection of potentially useful test compounds. Of the 23 pure compounds, only five (RL008, RL009, RL011, RL012 and RL013) had MIC values lower than $128 \mu \mathrm{g} / \mathrm{mL}$ in any tested isolates. These five compounds were particularly effective in suppressing M. abscessus. The MIC values for these compounds ranged from $<1$ to $>128 \mu \mathrm{g} / \mathrm{mL}$ (Table 2). The five effective pure compounds were selected for further analysis. No significant association was observed between colony morphotypes of M. abcessus isolates and their susceptibility to the test compounds and clarithromycin $(P$-value $>0.05$, Table S1).

\section{Toxicity testing on human RBCs and WBCs}

The hemolytic activity of the five selected compounds (RL008, RL009, RL011, RL012 and RL013) on human RBCs was evaluated. None showed any hemolytic effect on RBCs $(<1 \%)$ at the various concentrations used (at the compound concentration before adding RBC suspension: 1, 2, 4, 8, 16, 32, 64, and $128 \mu \mathrm{g} / \mathrm{mL}$ ) (Fig. 2 and Table S2). Indeed, the percentage of hemolysis was less than in the negative controls (RBC suspension in 1\% PBS that was used as the baseline). RBC morphologies under the light microscope were displayed as the cell shrinks

(Fig. 3). These findings indicate that the compounds were not harmful to human RBCs.

For WBCs, the percentage of dead cells following exposure to each tested compound is presented in Fig. 4 and Table S3, and the statistical analysis together with ANOVA with LSD 
253 post-hoc multiple comparisons revealed significantly differed among compounds (Fig. 4F). The

254 toxic effects were concentration-dependent. All five compounds caused death of $15-20 \%$ of cells

255 at the final concentrations of $64-128 \mu \mathrm{g} / \mathrm{mL}$, except for compound RL013, for which the

256 mortality rate was significantly lower (3-8\%). These results suggest that the compounds have a

257 low toxicity towards human WBCs.

258

259

Synergistic antimycobacterial activity of pure compounds and clarithromycin

Based on phenotypic results, fourteen M. abscessus isolates ( 7 isolates with inducible and

7 isolates with acquired resistance to clarithromycin) (Table 2) were randomly selected to study

262

the synergistic effect against these of five pure compounds in combination with clarithromycin.

Results (Table 3) showed that the highest degree of synergism was observed for the RL008/CLA and RL009/CLA combinations (FICI ranging from 0.13 to 0.50 ), which inhibited eight $M$.

abscessus isolates (57\%) (Fig. 1). The second strongest synergistic activity was observed for the

RL012/CLA combination, followed by the RL013/CLA combination, which showed synergistic effects against seven (50\%) and six isolates (42.9\%), respectively. The RL011/CLA combination showed the lowest synergistic effect, inhibiting only five isolates $(35.7 \%)$.

\section{Discussion}

272 of morbidity and mortality (Cassidy et al. 2009). The emergence, evolution and spread of $M$. abscessus infection is highly associated with drug resistance and treatment failure (Tung et al. 2015). Antibiotic resistance to M. abscessus is a major public health concern worldwide,

275 including in Thailand (Imwidthaya et al. 1990; Phowthongkum et al. 2005). Clarithromycin, a 
276 macrolide antibiotic, has a broad spectrum of antimicrobial activity that inhibits a range of

277 Gram-positive and Gram-negative microorganisms (Peters \& Clissold 1992). It is often a drug of

278 choice for the treatment of serious infections caused by M. abscessus. However, M. abscessus

279 clinical isolates with reduced susceptibility to clarithromycin have emerged, resulting in a

280 prolonged treatment course and poor clinical outcomes (Li et al. 2017). Clarithromycin

281 monotherapy is associated with treatment failure. A combination of antimicrobial agents may be

282 of therapeutic benefit and efficacious in the treatment of infections caused by clarithromycin-

283 resistant $M$. abscessus. There is therefore a need to search for new sources of antimycobacterial

284 substances. Plants produce a variety of bioactive compounds, sometimes with known therapeutic

285 properties (Rios \& Recio 2005). They are good sources of powerful antibiotic metabolites and

286 can treat various diseases (Hernandez-Garcia et al. 2019). This study was conducted to evaluate

287 the antimycobacterial activity of different secondary metabolites of plant origin against

288 clarithromycin-susceptible and -resistant M. abscessus isolates.

289 Researchers have isolated several such compounds and demonstrated their activities

290 against mycobacteria, including M. tuberculosis (Baldin et al. 2018; Chaipukdee et al. 2016;

291 Jimenez-Arellanes et al. 2013; Jyoti et al. 2016; Kanokmedhakul et al. 2012; Molina-Salinas et

292 al. 2006; Naik et al. 2014; Nkot et al. 2018). However, antimycobacterial activities of secondary

293 metabolites against M. abscessus have rarely been reported. We used 23 secondary metabolites

294 isolated from A. monophylla, P. filamentosa, A. conyzoides, R. wittii and N. nambi against M.

295 abscessus with different clarithromycin-resistance levels. The most effective compounds were

296 RL008, RL009, RL011, RL012, and RL013, which exhibited MIC values ranging from $<1$ to

$297>128 \mu \mathrm{g} / \mathrm{mL}$. 
Previous reports showed that colony morphology was not associated with susceptibility

299

300

301

302

303

304

305

306

307

308

309

310

311

312

313

314

315

316

317

318

319

320

to first-line antibiotics (Ruger et al. 2014). However, Clary et al. (2018) reported that certain colony morphotypes of $M$. abscessus were associated with biofilm formation and prolonged intracellular survival. We investigated the relationship between the colony morphotypes and the susceptibility to the compounds and clarithromycin. No significant association was found. This might be due to inadequate sample size. Therefore, such a relationship is still unconfirmed and requires further investigation.

Checking the toxicity of secondary metabolites on both human RBCs and WBCs is of importance when selecting candidates for antimycobacterial drugs. Our results demonstrate that the selected secondary metabolites are not harmful towards RBCs. No hemolysis was found among various concentrations of each compound. In fact, the degree of hemolysis in the presence of the test compounds was lower than in the negative controls (PBS), indicating the compounds may help to preserve RBCs better than the PBS control. The RBC shrinkage observed at high compound concentrations was an osmotic effect due to the extra-cellular concentrations. These results agree with those from a previous study (Lima Viana et al. 2018), which evaluated the antimicrobial activity of Bixa orellana secondary metabolites to treat Mycobacterium infections.

In our study, the secondary metabolites tested did not induce significant toxicity in human RBCs. The plant secondary metabolites that we tested killed $3 \%$ to $20 \%$ of WBCs at their MIC levels $(128 \mu \mathrm{g} / \mathrm{mL})$. RL013 had the lowest cytotoxicity to leukocytes with the fewest WBC deaths $(\approx 3 \%)$, lower than that caused by RL009 and RL012. Although toxicity for WBCs was quite high, these compounds nevertheless had potential to inhibit clarithromycin-resistant $M$. abscessus isolates. While chemical hair dye had similar toxicity for human WBCs and RBCs (Maiti et al. 2016), our plant-extracted compound had higher toxicity for WBCs than for RBCs. 
321 The ability of immune cells (i.e., WBCs) to absorb foreign compounds might be a possible

322 explanation (Keselowsky et al. 2020; Wang et al. 2018). Therefore, compounds with less toxicity

323 for leukocytes should be selected for further study to avoid affecting the host immune system.

324 In this study, a combination of the tested compounds and clarithromycin had synergistic

325 effects on some $M$. abscessus isolates with acquired or inducible clarithromycin resistance. No

326 antagonistic effect of combining these substances was found. Among the five effective

327 compounds tested, RL008 and RL009 proved to be the best in a combined treatment with

328 clarithromycin, frequently showing a synergistic effect, with the FICI values ranging from 0.13

329 to 0.5. Combined with RL008 and RL009, the average MICs of clarithromycin alone were

330 reduced up to 16 -fold (i.e., reduced from 512 to $32 \mu \mathrm{g} / \mathrm{mL}$ ). Both RL008 and RL009 had low

331 toxicity against RBCs and WBCs at the MIC levels. These results are consistent with those of

332 Rahgozar et al. (2018), who found that the best synergistic results against Mycobacterium bovis

333 were obtained for extracts of Lavandula stoechas and Datura stramonium in combination with

334 ethambutol. In addition, Lopes et al. (2014) reported that a synergism was observed against $M$.

335 tuberculosis with eupomatenoid-5 (EUP-5), extracted from Piper solmsianum C. DC. var.

336 solmsianum plus rifampicin, and EUP-5 plus ethambutol combinations. Similar observations of

337 synergism between plant secondary metabolites and various drugs against mycobacteria have

338 been reported in other studies (Aro et al. 2016; Mossa et al. 2004; Naik et al. 2014). We observed

339 that synergistic effects of combining the test compounds with clarithromycin occurred against $M$.

340 abscessus isolates with inducible as well as acquired clarithromycin resistance. Therefore, plant

341 secondary metabolites could be used for treatment of both forms of resistance. However, the

342 association between antimycobacterial susceptibility and clarithromycin-resistance type remains

343 unclear. 
health concern. Although our combinations of pure compounds and clarithromycin did not

abscessus isolates (showing either inducible or acquired resistance) were inhibited. This infection.

(Comini et al. 2011; Kemegne et al. 2017; Lu et al. 2011; Xu et al. 2017). investigated in vivo for more conclusive results. The relationship between the colony morphotype of $M$. abcessus isolates and their susceptibility to the combination of compounds and clarithromycin is required. Further work is necessary using structural variants of the plant secondary metabolites identified here to improve their antimycobacterial efficacy. Additional studies might also evaluate antifungal, antiviral and antiparasitic activities of these compounds.

\section{Conclusion}

We report that five compounds isolated from medicinal plants have potent 
366 acceptable results in toxicity tests towards RBCs and WBCs. These compounds might be used as

367 an alternative treatment and should be further studied to develop anti-tuberculous drugs.

368

369 Acknowledgments

370 We would like to acknowledge Prof. David Blair for editing the MS via Publication Clinic KKU, 371 Thailand.

372

373

\section{References}

374

375

376

377

Ahond A, Guilhem J, Hamon J, Hurtado J, Poupat C, Pusset J, Pusset M, Sévenet T, and Potier P. 1990. Bubbialine et Bubbialidine, Alcaloïdes Nouveaux Extraits de Zygogynum pauciflorum. J Nat Prod 53:875-881. 10.1021/np50070a015

Ananta P, Kham-Ngam I, Chetchotisakd P, Chaimanee P, Reechaipichitkul W, Namwat W, Lulitanond V, and Faksri K. 2018. Analysis of drug-susceptibility patterns and gene sequences associated with clarithromycin and amikacin resistance in serial Mycobacterium abscessus isolates from clinical specimens from Northeast Thailand. PLoS One 13:e0208053. 10.1371/journal.pone.0208053

Andrews S. 2010. FastQC: A Quality Control Tool for High Throughput Sequence Data [Online]. Available online at: http://www.bioinformatics.babraham.ac.uk/projects/fastqc/ (access May 2021).

Aro AO, Dzoyem JP, Eloff JN, and McGaw LJ. 2016. Extracts of six Rubiaceae species combined with rifampicin have good in vitro synergistic antimycobacterial activity and good anti-inflammatory and antioxidant activities. BMC Complement Altern Med 16:385. 10.1186/s12906-016-1355-y

PeerJ reviewing PDF | (2021:07:63417:1:2:NEW 3 Oct 2021) 
Baldin VP, Scodro RBL, Lopes-Ortiz MA, de Almeida AL, Gazim ZC, Ferarrese L, Faioes VDS, Torres-Santos EC, Pires CTA, Caleffi-Ferracioli KR, Siqueira VLD, Cortez DAG, and Cardoso RF. 2018. Anti-Mycobacterium tuberculosis activity of essential oil and 6,7dehydroroyleanone isolated from leaves of Tetradenia riparia (Hochst.) Codd (Lamiaceae). Phytomedicine 47:34-39. 10.1016/j.phymed.2018.04.043

Bolger AM, Lohse M, and Usadel B. 2014. Trimmomatic: a flexible trimmer for Illumina sequence data. Bioinformatics 30:2114-2120. 10.1093/bioinformatics/btu170

Boyum A. 1976. Isolation of lymphocytes, granulocytes and macrophages. Scand J Immunol Suppl 5:9-15. 10.1111/j.1365-3083.1976.tb03851.x

Cassidy PM, Hedberg K, Saulson A, McNelly E, and Winthrop KL. 2009. Nontuberculous mycobacterial disease prevalence and risk factors: a changing epidemiology. Clin Infect Dis 49:e124-129. 10.1086/648443

Chaipukdee N, Kanokmedhakul K, Kanokmedhakul S, Lekphrom R, and Pyne SG. 2016. Two new bioactive iridoids from Rothmannia wittii. Fitoterapia 113:97-101. 10.1016/j.fitote.2016.07.007

Clary G, Sasindran SJ, Nesbitt N, Mason L, Cole S, Azad A, McCoy K, Schlesinger LS, and Hall-Stoodley L. 2018. Mycobacterium abscessus smooth and rough morphotypes form antimicrobial-tolerant biofilm phenotypes but are killed by acetic acid. Antimicrob Agents Chemother 62. 10.1128/AAC.01782-17

CLSI. 2018. Susceptibility testing of mycobacteria, Nocardia spp., and other aerobic actinomycetes, 3rd ed, CLSI standard document M24. Clinical and Laboratory Standards Institute, Wayne, PA. 
411 Comini LR, Montoya SC, Paez PL, Arguello GA, Albesa I, and Cabrera JL. 2011. Antibacterial

412

413

414

415

416

417

418

419

420

421

422

423

424

425

426

427

428

429

430

431

432

433

activity of anthraquinone derivatives from Heterophyllaea pustulata (Rubiaceae). J

Photochem Photobiol B 102:108-114. 10.1016/j.jphotobiol.2010.09.009

De Almeida IN, Da Silva Carvalho W, Rossetti ML, Costa ER, and De Miranda SS. 2013.

Evaluation of six different DNA extraction methods for detection of Mycobacterium tuberculosis by means of PCR-IS6110: preliminary study. BMC Res Notes 6:561.

10.1186/1756-0500-6-561

Doern CD. 2014. When does 2 plus 2 equal 5? A review of antimicrobial synergy testing. J Clin Microbiol 52:4124-4128. 10.1128/JCM.01121-14

Garcia LS. 2010. Synergism testing: broth microdilution checkerboard and broth macrodilution methods. In Clinical microbiology procedures handbook, 3rd ed and 2007 update: ASM Press, Washington, DC.

Hernandez-Garcia E, Garcia A, Garza-Gonzalez E, Avalos-Alanis FG, Rivas-Galindo VM, Rodriguez-Rodriguez J, Alcantar-Rosales VM, Delgadillo-Puga C, and Del Rayo Camacho-Corona M. 2019. Chemical composition of Acacia farnesiana (L) wild fruits and its activity against Mycobacterium tuberculosis and dysentery bacteria. $J$ Ethnopharmacol 230:74-80. 10.1016/j.jep.2018.10.031

Imwidthaya P, Komolpis P, Suthiravitayavaniz K, and Rienthong S. 1990. In vitro drug susceptibility of Mycobacterium other than tubercle bacilli. J Med Assoc Thai 73:438442.

Iroh Tam PY, Kline S, Ward G, and Ferrieri P. 2015. Non-tuberculous mycobacterial infection in hospitalized children: a case series. Epidemiol Infect 143:3173-3181. $10.1017 / \mathrm{S} 0950268815000333$ 
434 Jimenez-Arellanes A, Luna-Herrera J, Ruiz-Nicolas R, Cornejo-Garrido J, Tapia A, and Yepez-

435 Mulia L. 2013. Antiprotozoal and antimycobacterial activities of Persea americana

436 seeds. BMC Complement Altern Med 13:109. 10.1186/1472-6882-13-109

437

438

439

440

441

442

443

444

445

446

447

448

449

450

451

452

453

454

455

456

Jyoti MA, Nam KW, Jang WS, Kim YH, Kim SK, Lee BE, and Song HY. 2016. Antimycobacterial activity of methanolic plant extract of Artemisia capillaris containing ursolic acid and hydroquinone against Mycobacterium tuberculosis. J Infect Chemother 22:200-208. 10.1016/j.jiac.2015.11.014

Kaewpiboon C, Lirdprapamongkol K, Srisomsap C, Winayanuwattikun P, Yongvanich T, Puwaprisirisan P, Svasti J, and Assavalapsakul W. 2012. Studies of the in vitro cytotoxic, antioxidant, lipase inhibitory and antimicrobial activities of selected Thai medicinal plants. BMC Complement Altern Med 12:217. 10.1186/1472-6882-12-217

Kanokmedhakul S, Lekphrom R, Kanokmedhakul K, Hahnvajanawong C, Bua-art S, Saksirirat W, Prabpai S, and Kongsaeree P. 2012. Cytotoxic sesquiterpenes from luminescent mushroom Neonothopanus nambi. Tetrahedron 68:8261-8266. 10.1016/j.tet.2012.07.057

Kemegne GA, Mkounga P, Essia Ngang JJ, Sado Kamdem SL, and Nkengfack AE. 2017. Antimicrobial structure activity relationship of five anthraquinones of emodine type isolated from Vismia laurentii. BMC Microbiol 17:41. 10.1186/s12866-017-0954-1

Keselowsky BG, Acharya A, and Lewis JS. 2020. 2.2.3 - Innate and Adaptive Immunity: The Immune Response to Foreign Materials. In: Wagner WR, Sakiyama-Elbert SE, Zhang G, and Yaszemski MJ, eds. Biomaterials Science (Fourth Edition): Academic Press, 747775.

Kham-Ngam I, Chetchotisakd P, Ananta P, Chaimanee P, Reechaipichitkul W, Lulitanond V, Namwat W, and Faksri K. 2019. Differentiation between persistent infection/colonization 
457

458

459

460

461

462

463

464

465

466

467

468

469

470

471

472

473

474

475

476

477

478

and re-infection/re-colonization of Mycobacterium abscessus isolated from patients in Northeast Thailand. Infect Genet Evol 68:35-42. 10.1016/j.meegid.2018.12.001

Kham-Ngam I, Chetchotisakd P, Ananta P, Chaimanee P, Sadee P, Reechaipichitkul W, and Faksri K. 2018. Epidemiology of and risk factors for extrapulmonary nontuberculous mycobacterial infections in Northeast Thailand. PeerJ 6:e5479. 10.7717/peerj.5479

Li B, Yang S, Chu H, Zhang Z, Liu W, Luo L, Ma W, and Xu X. 2017. Relationship between antibiotic susceptibility and genotype in Mycobacterium abscessus clinical isolates. Front Microbiol 8:1739. 10.3389/fmicb.2017.01739

Li H. 2013. Aligning sequence reads, clone sequences and assembly contigs with BWA-MEM. $\operatorname{arXiv.~}$

Li H, Handsaker B, Wysoker A, Fennell T, Ruan J, Homer N, Marth G, Abecasis G, Durbin R, and Proc GPD. 2009. The Sequence Alignment/Map format and SAMtools. Bioinformatics 25:2078-2079. 10.1093/bioinformatics/btp352

Lima Viana J, Zagmignan A, Lima Lobato LF, Gomes Abreu A, Jr., da Silva LCN, de Sa JC, Monteiro CA, Lago JHG, Goncalves LM, Carvalho RC, Neto LGL, and de Sousa EM. 2018. Hydroalcoholic extract and ethyl acetate fraction of Bixa orellana leaves decrease the inflammatory response to Mycobacterium abscessus subsp. massiliense. Evid Based Complement Alternat Med 2018:6091934. 10.1155/2018/6091934

Lopes MA, Ferracioli KR, Siqueira VL, de Lima Scodro RB, Cortez DA, da Silva RZ, and Cardoso RF. 2014. In vitro interaction of eupomatenoid-5 from Piper solmsianum C. DC. var. solmsianum and anti-tuberculosis drugs. Int J Tuberc Lung Dis 18:1513-1515. 10.5588/ijtld.14.0229

Peer) reviewing PDF | (2021:07:63417:1:2:NEW 3 Oct 2021) 
479 Lu C, Wang H, Lv W, Xu P, Zhu J, Xie J, Liu B, and Lou Z. 2011. Antibacterial properties of

480

481

482

483

484

485

486

487

488

489

490

491

492

493

494

495

496

497

498

499

500

501

anthraquinones extracted from rhubarb against Aeromonas hydrophila. Fisheries Science 77:375. 10.1007/s12562-011-0341-z

Maiti S, Sasmal K, Sinha SS, and Singh M. 2016. Analysis of cytotoxicity and genotoxicity on E. coli, human blood cells and Allium cepa suggests a greater toxic potential of hair dye. Ecotoxicol Environ Saf 124:248-254. 10.1016/j.ecoenv.2015.10.028

McKenna A, Hanna M, Banks E, Sivachenko A, Cibulskis K, Kernytsky A, Garimella K, Altshuler D, Gabriel S, Daly M, and DePristo MA. 2010. The Genome Analysis Toolkit: a MapReduce framework for analyzing next-generation DNA sequencing data. Genome Res 20:1297-1303. 10.1101/gr.107524.110

Molina-Salinas GM, Ramos-Guerra MC, Vargas-Villarreal J, Mata-Cardenas BD, BecerrilMontes P, and Said-Fernandez S. 2006. Bactericidal activity of organic extracts from Flourensia cernua DC against strains of Mycobacterium tuberculosis. Arch Med Res 37:45-49. 10.1016/j.arcmed.2005.04.010

Mossa JS, El-Feraly FS, and Muhammad I. 2004. Antimycobacterial constituents from Juniperus procera, Ferula communis and Plumbago zeylanica and their in vitro synergistic activity with isonicotinic acid hydrazide. Phytother Res 18:934-937. 10.1002/ptr.1420

Naik SK, Mohanty S, Padhi A, Pati R, and Sonawane A. 2014. Evaluation of antibacterial and cytotoxic activity of Artemisia nilagirica and Murraya koenigii leaf extracts against mycobacteria and macrophages. BMC Complement Altern Med 14:87. 10.1186/14726882-14-87

Nessar R, Cambau E, Reyrat JM, Murray A, and Gicquel B. 2012. Mycobacterium abscessus: a new antibiotic nightmare. J Antimicrob Chemother 67:810-818. 10.1093/jac/dkr578

Peer] reviewing PDF | (2021:07:63417:1:2:NEW 3 Oct 2021) 
502 Nkot JL, Ngono Bikobo DS, Abouem AZA, Nyemeck NM, 2nd, Moni Ndedi EDF, Betote 503 Diboue PH, Pegnyemb DE, Bochet CG, and Koert U. 2018. Antitubercular evaluation of

504

505

506

507

508

509

510

511

512

513

514

515

516

517

518

519

520

521

522

523 root extract and isolated phytochemicals from Lophira lanceolata against two resistant strains of Mycobacterium tuberculosis. Pharm Biol 56:318-324.

$10.1080 / 13880209.2018 .1476559$

Pailee P, Prawat H, Ploypradith P, Mahidol C, Ruchirawat S, and Prachyawarakorn V. 2020. Atalantiaphyllines A-G, prenylated acridones from Atalantia monophylla DC. and their aromatase inhibition and cytotoxic activities. Phytochemistry 180:112525. 10.1016/j.phytochem.2020.112525

Peters DH, and Clissold SP. 1992. Clarithromycin. A review of its antimicrobial activity, pharmacokinetic properties and therapeutic potential. Drugs 44:117-164. 10.2165/00003495-199244010-00009

Phowthongkum P, Prasanthai V, Udomsantisook N, and Suankratay C. 2005. Rapidly growing mycobacteria in King Chulalongkorn Memorial Hospital and review of the literature in Thailand. J Med Assoc Thai 88:1153-1162.

Qasim M, Wang BH, Zou HS, Lin YW, Dash CK, Bamisile BS, Hussai M, Zhao ZW, and Wang LD. 2018. Phylogenetic relationship and genetic diversity of citrus psyllid populations from China and Pakistan and their associated Candidatus bacterium. Mol Phylogenet Evol 126:173-180. 10.1016/j.ympev.2018.04.028

Rahgozar N, Bakhshi Khaniki G, and Sardari S. 2018. Evaluation of antimycobacterial and synergistic activity of plants selected based on cheminformatic parameters. Iran Biomed J 22:401-407. 10.29252/.22.6.401 
524 Rios JL, and Recio MC. 2005. Medicinal plants and antimicrobial activity. J Ethnopharmacol

525

526

527

528

529

530

531

532

533

534

535

536

537

538

539

540

541

542

543

544

$$
\text { 100:80-84. 10.1016/j.jep.2005.04.025 }
$$

Ruger K, Hampel A, Billig S, Rucker N, Suerbaum S, and Bange FC. 2014. Characterization of rough and smooth morphotypes of Mycobacterium abscessus isolates from clinical specimens. J Clin Microbiol 52:244-250. 10.1128/JCM.01249-13

Sombatsri A, Thummanant Y, Sribuhom T, Boonmak J, Youngme S, Phusrisom S, Kukongviriyapan V, and Yenjai C. 2018. New limonophyllines A-C from the stem of Atalantia monophylla and cytotoxicity against cholangiocarcinoma and HepG2 cell lines. Arch Pharm Res 41:431-437. 10.1007/s12272-018-1021-7

Strober W. 2015. Trypan blue exclusion test of cell viability. Curr Protoc Immunol 111:A3 B 1A3 B 3. 10.1002/0471142735.ima03bs111

Sukari M. A NBK, Lajis N. H, Ee G. C. L, Rahmani M, Ahmad F. B. H, Yusof U. K. 2004. Chemical constituents of Kaempferia angustifolia (Zingiberaceae). Orient J Chem 20.

Tung YJ, Bittaye SO, Tsai JR, Lin CY, Huang CH, Chen TC, Lin WR, Chang K, Lai CC, Lu PL, and Chen YH. 2015. Risk factors for microbiologic failure among Taiwanese adults with Mycobacterium abscessus complex pulmonary disease. J Microbiol Immunol Infect 48:437-445. 10.1016/j.jmii.2014.08.005

Velayati AA, Farnia P, Mozafari M, Malekshahian D, Seif S, Rahideh S, and Mirsaeidi M. 2014. Molecular epidemiology of nontuberculous mycobacteria isolates from clinical and environmental sources of a metropolitan city. PLoS One 9:e114428. 10.1371/journal.pone.0114428

Peer] reviewing PDF | (2021:07:63417:1:2:NEW 3 Oct 2021) 
545 Wang K, Conlon M, Ren W, Chen BB, and Bączek T. 2018. Natural products as targeted

546

547

548

549

550

551

552

553

554

555

556

557

558

559

560

modulators of the immune system. Journal of Immunology Research 2018:7862782. $10.1155 / 2018 / 7862782$

Wang XF, Chen JY, and Lu WJ. 1985. Studies on the chemical constituents of Knoxia valerianoides Thorel ex Pitard. Yao Xue Xue Bao 20:615-618.

Wisetsai A, Lekphrom R, and Schevenels FT. 2021. New anthracene and anthraquinone metabolites from Prismatomeris filamentosa and their antibacterial activities. Nat Prod Res 35:1582-1589. 10.1080/14786419.2019.1627352

Wisetsai A, Schevenels FT, Faksri K, Tontapha S, Amornkitbamrung V, and Lekphrom R. 2020. New iridoid glucosides from the roots of Rothmannia wittii (Craib) Bremek. Nat Prod Res:1-9. 10.1080/14786419.2020.1813133

Xu L, Li X, Cui Y, Pang M, Wang F, and Qi J. 2017. Antibacterial activity of anthraquinone from aloe on spiced pig head. IOP Conference Series: Materials Science and Engineering 275:012014. 10.1088/1757-899x/275/1/012014

Yim SH, Kim HJ, and Lee IS. 2003. A polyacetylene and flavonoids from Cirsium rhinoceros. Archives of Pharmacal Research 26:128-131. 10.1007/BF02976657 
561 Table 1 Compounds used in this study.

\begin{tabular}{|c|c|c|c|c|}
\hline No.* & Code & Compound & Source & References \\
\hline 1 & RL001 & $N$-methylcycloatalaphylline A & Roots of A. monophylla & (Pailee et al., 2020) \\
\hline 2 & RL006 & yukocitrine & Roots of A. monophylla & (Pailee et al., 2020) \\
\hline 3 & RL002 & $\mathrm{N}$-methylatalaphylline & Roots of A. monophylla & (Pailee et al., 2020) \\
\hline 4 & RL007 & atalaphylline & Roots of A. monophylla & (Pailee et al., 2020) \\
\hline 5 & RL004 & atalaphylline-3,5-dimethyl ether & Roots of A. monophylla & (Pailee et al., 2020) \\
\hline 6 & RL003 & 2,2-dimethylchromenocoumarin & Roots of A. monophylla & (Pailee et al., 2020) \\
\hline 7 & RL005 & auraptene & Roots of A. monophylla & (Pailee et al., 2020) \\
\hline 8 & RL009 & rubiadin-1-methyl ether & Roots of $P$. filamentosa & (Wisetsai et al., 2021) \\
\hline 9 & RL010 & rubiadin & Roots of $P$. filamentosa & (Wisetsai et al., 2021) \\
\hline 10 & RL011 & knoxiadin & Roots of $P$. filamentosa & (Wang et al., 1985) \\
\hline 11 & RL008 & nordamnacanthal & Roots of $P$. filamentosa & (Wisetsai et al., 2021) \\
\hline 12 & RL012 & damnacanthal & Roots of $P$. filamentosa & (Wisetsai et al., 2021) \\
\hline 13 & RL013 & damnacanthol & Roots of $P$. filamentosa & (Wisetsai et al., 2021) \\
\hline 14 & RL014 & 3',4',7-tri- $O$-methylluteolin & Flowers of $A$. conyzoides & (Ahond et al., 1990) \\
\hline 15 & RL015 & 4',7-di-O-methylapigenin & Flowers of $A$. conyzoides & (Ahond et al., 1990) \\
\hline 16 & RL016 & 4'-O-methylapigenin & Flowers of $A$. conyzoides & (Yim et al., 2003) \\
\hline 17 & \multicolumn{2}{|c|}{ RL017 2'-hydroxy-4,4',6'-trimethoxychalcone } & Flowers of $A$. conyzoides & (Sukari M. A, 2004) \\
\hline 18 & RL020 & 3,5-dihydroxycinnamate & Roots of $R$. wittii & (Wisetsai et al., 2020) \\
\hline 19 & RL021 & lippianoside B & Roots of $R$. wittii & (Wisetsai et al., 2020) \\
\hline 20 & RL022 & rothmannioside $\mathrm{C}$ & Roots of $R$. wittii & (Wisetsai et al., 2020) \\
\hline 21 & RL023 & rothmannioside A & Roots of $R$. wittii & (Wisetsai et al., 2020) \\
\hline 22 & RL024 & rothmannioside B & Roots of $R$. wittii & (Wisetsai et al., 2020) \\
\hline 23 & RL019 & aurisin $\mathrm{A}$ & Cultured mycelium of $N$. nam & (Kanokmedhakul et al., 2012) \\
\hline
\end{tabular}

562 Note: Compounds 1-22 were isolated from four medicinal plants (Atalantia monophylla,

563 Prismatomeris filamentosa, Ageratum conyzoides, and Rothmannia wittii). Compound 23 was

564 isolated from the cultured mycelium of the luminescent mushroom Neonothopanus nambi. 
566 Table 2 Antimycobacterial activity screening of five plant secondary metabolites against 30 M. abscessus isolates.

\begin{tabular}{|c|c|c|c|c|c|c|c|c|c|c|c|c|c|c|}
\hline \multirow[t]{2}{*}{ Isolates } & \multirow[t]{2}{*}{ Organism } & \multirow[t]{2}{*}{$\begin{array}{c}\begin{array}{c}\text { Colony } \\
\text { morphology }\end{array} \\
\end{array}$} & \multicolumn{7}{|c|}{ MIC value of clarithromycin $(\mu \mathrm{g} / \mathrm{mL})$} & \multicolumn{5}{|c|}{$\begin{array}{c}\begin{array}{c}\text { Antimycobacterial screening against } \\
\text { M. abscessus isolates }\end{array} \\
\text { MIC value of pure compounds }(\mu \mathrm{g} / \mathrm{mL})\end{array}$} \\
\hline & & & MIC & Phenotype & MIC & Phenotype & MIC & Phenotype & DST interpretation & RL008 & RL009 & RL011 & RL012 & RL013 \\
\hline 80097 & M. abscessus subsp. abscessus & Mixed & 0.25 & Susceptible & 8 & Resistant & 16 & Resistant & Inducible & 128 & 16 & 64 & 64 & 128 \\
\hline 80167 & M. abscessus subsp. massiliense & Rough & $\geq 16$ & Resistant & $\geq 16$ & Resistant & $\geq 16$ & Resistant & Acquired & 128 & 8 & 64 & 64 & 128 \\
\hline 80225 & M. abscessus subsp. abscessus & Rough & 0.25 & Susceptible & 4 & Intermediate & $\geq 16$ & Resistant & Inducible & $<1$ & $<1$ & $<1$ & $<1$ & $<1$ \\
\hline 80448 & M. abscessus subsp. massiliense & Rough & 0.12 & Susceptible & 0.25 & Susceptible & 2 & Susceptible & Susceptible & 128 & 128 & 128 & 128 & 128 \\
\hline 80524 & M. abscessus subsp. massiliense & Mixed & 0.5 & Susceptible & 2 & Susceptible & 2 & Susceptible & Susceptible & 128 & 128 & 128 & 128 & 128 \\
\hline 80700 & M. abscessus subsp. massiliense & Rough & $>16$ & Resistant & $>16$ & Resistant & $>16$ & Resistant & Acquired & $>128$ & 128 & 64 & $>128$ & 128 \\
\hline 80824 & M. abscessus subsp. massiliense & Mixed & 0.25 & Susceptible & 0.5 & Susceptible & 1 & Susceptible & Susceptible & 128 & 16 & 32 & 128 & 128 \\
\hline 80838 & M. abscessus subsp. abscessus & Mixed & 1 & Susceptible & 16 & Resistant & 16 & Resistant & Inducible & 128 & 128 & 128 & 128 & $>128$ \\
\hline 80866 & M. abscessus subsp. massiliense & Smooth & 0.12 & Susceptible & 0.12 & Susceptible & $>16$ & Resistant & Inducible & 128 & 128 & 128 & 128 & 128 \\
\hline 80901 & M. abscessus subsp. abscessus & Rough & 1 & Susceptible & 8 & Resistant & 16 & Resistant & Inducible & $>128$ & 128 & 128 & 128 & $>128$ \\
\hline 80988 & M. abscessus subsp. abscessus & Rough & 0.12 & Susceptible & 0.25 & Susceptible & 16 & Resistant & Inducible & 128 & $>128$ & 64 & 128 & $>128$ \\
\hline 81103 & M. abscessus subsp. abscessus & Smooth & 2 & Susceptible & 2 & Susceptible & 16 & Resistant & Inducible & 128 & 128 & 64 & 128 & 128 \\
\hline 81350 & M. abscessus subsp. massiliense & Rough & $\leq 0.06$ & Susceptible & 0.12 & Susceptible & 0.12 & Susceptible & Susceptible & $>128$ & 128 & 128 & 128 & 128 \\
\hline 81422 & M. abscessus subsp. abscessus & Rough & 0.5 & Susceptible & 1 & Susceptible & $\geq 16$ & Resistant & Inducible & $>128$ & 128 & 128 & 128 & $>128$ \\
\hline 81463 & M. abscessus subsp. massiliense & Smooth & $\leq 0.06$ & Susceptible & 0.12 & Susceptible & 0.25 & Susceptible & Susceptible & 128 & 128 & 64 & 128 & 128 \\
\hline 81499 & M. abscessus subsp. massiliense & Rough & 16 & Resistant & 16 & Resistant & 16 & Resistant & Acquired & 128 & 128 & 64 & 128 & 128 \\
\hline 81618 & M. abscessus subsp. abscessus & Mixed & 16 & Resistant & 16 & Resistant & 16 & Resistant & Acquired & 128 & 16 & 32 & 128 & 128 \\
\hline 81652 & M. abscessus subsp. massiliense & Mixed & 0.12 & Susceptible & 0.25 & Susceptible & 1 & Susceptible & Susceptible & 128 & 8 & 128 & 64 & 128 \\
\hline 81702 & M. abscessus subsp. massiliense & Rough & $>16$ & Resistant & $>16$ & Resistant & $>16$ & Resistant & Acquired & $>128$ & 128 & 64 & $>128$ & 128 \\
\hline 81838 & M. abscessus subsp. massiliense & Rough & 16 & Resistant & 16 & Resistant & $\geq 16$ & Resistant & Acquired & 128 & 128 & 128 & 128 & 128 \\
\hline 82119 & M. abscessus subsp. abscessus & Smooth & 2 & Susceptible & 16 & Resistant & 16 & Resistant & Inducible & 128 & 16 & 64 & 64 & 128 \\
\hline 82154 & M. abscessus subsp. abscessus & Smooth & 0.5 & Susceptible & 8 & Resistant & 16 & Resistant & Inducible & 128 & 32 & 64 & 64 & 128 \\
\hline 82593 & M. abscessus subsp. massiliense & Rough & $\leq 0.06$ & Susceptible & 0.12 & Susceptible & 0.5 & Susceptible & Susceptible & $>128$ & 128 & 128 & $>128$ & $>128$ \\
\hline
\end{tabular}




\begin{tabular}{|c|c|c|c|c|c|c|c|c|c|c|c|c|c|c|}
\hline 82895 & M. abscessus subsp. massiliense & Rough & 0.25 & Susceptible & 0.5 & Susceptible & 2 & Susceptible & Susceptible & 128 & $>128$ & 32 & 128 & $>128$ \\
\hline 82998 & M. abscessus subsp. abscessus & Smooth & 16 & Resistant & 16 & Resistant & 16 & Resistant & Acquired & 128 & 128 & 128 & 128 & 128 \\
\hline 83305 & M. abscessus subsp. abscessus & Smooth & 16 & Resistant & 16 & Resistant & 16 & Resistant & Acquired & $>128$ & 128 & 128 & 128 & 128 \\
\hline 83310 & M. abscessus subsp. abscessus & Rough & 8 & Resistant & 8 & Resistant & $\geq 16$ & Resistant & Acquired & 128 & $>128$ & 128 & $>128$ & $>128$ \\
\hline 83347 & M. abscessus subsp. massiliense & Mixed & 0.25 & Susceptible & 0.5 & Susceptible & 0.5 & Susceptible & Susceptible & 128 & 128 & 128 & 128 & $>128$ \\
\hline 90919 & M. abscessus subsp. massiliense & Mixed & 0.12 & Susceptible & 0.12 & Susceptible & 0.5 & Susceptible & Susceptible & 128 & 128 & 128 & 128 & $>128$ \\
\hline 826492 & M. abscessus subsp. massiliense & Mixed & 0.12 & Susceptible & 0.25 & Susceptible & 16 & Resistant & Inducible & 128 & 16 & 64 & 64 & 128 \\
\hline
\end{tabular}

567 Note: MIC, minimum inhibitory concentration; DST, drug susceptibility testing; Acquired, acquired resistance; Inducible, inducible

568 resistance. 
569 Table 3 Synergistic activity of five plant secondary metabolites combined with clarithromycin against 14 clarithromycin-resistant $M$.

570 abscessus isolates.

\begin{tabular}{|c|c|c|c|c|c|c|c|c|c|c|c|c|c|c|c|c|c|}
\hline \multirow[b]{2}{*}{ Isolates } & \multirow[b]{2}{*}{$\begin{array}{c}\text { Susceptibility } \\
\text { profile }\end{array}$} & \multicolumn{6}{|c|}{ Individual MIC $(\mu \mathrm{g} / \mathrm{mL})$} & \multicolumn{5}{|c|}{ Combination MIC $(\mu \mathrm{g} / \mathrm{mL})$} & \multicolumn{5}{|c|}{ FICI } \\
\hline & & 己 & $\stackrel{\infty}{\stackrel{0}{1}}$ & $\underset{\hat{\theta}}{\underline{a}}$ & $\underset{\overline{1}}{\bar{a}}$ & $\underset{\mathfrak{a}}{\mathfrak{a}}$ & $\stackrel{m}{a}$ & 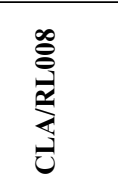 & 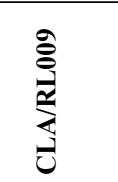 & 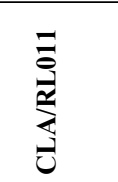 & 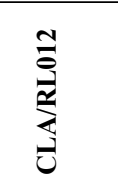 & 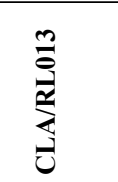 & 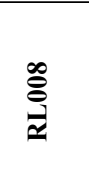 & 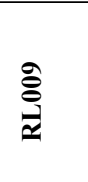 & $\underset{\overline{3}}{\bar{a}}$ & $\underset{\mathfrak{a}}{\mathfrak{a}}$ & $\stackrel{m}{\bar{a}}$ \\
\hline 81499 & Acquired & 64 & 128 & 128 & 64 & 128 & 128 & $4 / 16$ & $32 / 128$ & $64 / 128$ & $4 / 8$ & $16 / 64$ & 0.19 & 1.50 & 2.00 & 0.13 & 0.75 \\
\hline 82998 & Acquired & 16 & 128 & 128 & 128 & 128 & 128 & $8 / 32$ & $2 / 32$ & $8 / 64$ & $8 / 128$ & $8 / 32$ & 0.75 & 0.38 & 1.00 & 1.50 & 0.75 \\
\hline 83305 & Acquired & 16 & $>128$ & 128 & 128 & 128 & 128 & $16 / 128$ & $2 / 128$ & $16 / 128$ & $4 / 128$ & $4 / 64$ & 2.00 & 1.13 & 2.00 & 1.25 & 0.75 \\
\hline 83310 & Acquired & 4 & 128 & $>128$ & 128 & $>128$ & $>128$ & $1 / 8$ & $2 / 8$ & $1 / 8$ & $4 / 8$ & $1 / 8$ & 0.31 & 0.56 & 0.31 & 1.06 & 0.31 \\
\hline 81618 & Acquired & 64 & 128 & 16 & 32 & 128 & 128 & $64 / 128$ & $32 / 64$ & $64 / 128$ & $64 / 128$ & $64 / 128$ & 2.00 & 1.00 & 2.00 & 2.00 & 2.00 \\
\hline 80700 & Acquired & 512 & $>128$ & 128 & 64 & $>128$ & 128 & $512 / 128$ & $512 / 128$ & $512 / 128$ & $512 / 128$ & $512 / 128$ & 2.00 & 2.00 & 2.00 & 2.00 & 2.00 \\
\hline 81838 & Acquired & 16 & 128 & 128 & 128 & 128 & 128 & $1 / 8$ & $1 / 8$ & $1 / 8$ & $1 / 8$ & $1 / 8$ & 0.13 & 0.13 & 0.13 & 0.13 & 0.13 \\
\hline 82119 & Inducible & 512 & 128 & 16 & 64 & 64 & 128 & $32 / 8$ & $512 / 128$ & $512 / 64$ & $64 / 128$ & $512 / 64$ & 0.50 & 2.00 & 1.50 & 1.13 & 1.5 \\
\hline 80097 & Inducible & 512 & 128 & 16 & 64 & 64 & 128 & $32 / 8$ & $64 / 32$ & $32 / 64$ & $64 / 32$ & $128 / 64$ & 0.13 & 0.38 & 0.56 & 0.38 & 0.75 \\
\hline 80838 & Inducible & 128 & 128 & 128 & 128 & 128 & $>128$ & $32 / 8$ & $32 / 16$ & $64 / 8$ & $16 / 16$ & $16 / 32$ & 0.31 & 0.38 & 0.56 & 0.25 & 0.38 \\
\hline 80988 & Inducible & 4 & 128 & $>128$ & 64 & 128 & $>128$ & $2 / 8$ & $1 / 32$ & $1 / 8$ & $2 / 8$ & $2 / 8$ & 0.56 & 0.50 & 0.31 & 0.56 & 0.56 \\
\hline 80901 & Inducible & 16 & $>128$ & 128 & 128 & 128 & $>128$ & $4 / 8$ & $4 / 8$ & $4 / 8$ & $4 / 8$ & $4 / 16$ & 0.31 & 0.31 & 0.31 & 0.31 & 0.38 \\
\hline 82154 & Inducible & 32 & 128 & 32 & 64 & 64 & 128 & $4 / 16$ & $8 / 16$ & $8 / 16$ & $8 / 32$ & $8 / 16$ & 0.25 & 0.38 & 0.38 & 0.50 & 0.38 \\
\hline 81103 & Inducible & 64 & 128 & 128 & 64 & 128 & 128 & $32 / 8$ & $16 / 32$ & $16 / 128$ & $8 / 32$ & $16 / 32$ & 0.56 & 0.50 & 1.25 & 0.38 & 0.50 \\
\hline $\begin{array}{l}\text { Number } \\
\text { synerg }\end{array}$ & $\begin{array}{l}\text { isolates with } \\
\text { activity (\%) }\end{array}$ & & & & & & & & & & & & $8(57)$ & $8(57)$ & $\begin{array}{c}5 \\
(35.7)\end{array}$ & $7(50)$ & $\begin{array}{c}6 \\
(42.9)\end{array}$ \\
\hline
\end{tabular}


571 Note: MIC, minimum inhibitory concentration; CLA, clarithromycin; Acquired, acquired resistance; Inducible, inducible resistance;

572 FICI, fractional inhibitory concentration index

573 FICI interpretation: $\leq 0.5$ : synergy; $>0.5-1.0$ : additive; $>1.0-4.0$ : indifference; $>4.0$ : antagonism (Doern 2014)

574 Grey-shaded boxes shows synergistic effect

575 


\section{Table $\mathbf{1}$ (on next page)}

Compounds used in this study.

Note: Compounds 1-22 were isolated from four medicinal plants ( Atalantia monophylla, Prismatomeris filamentosa, Ageratum conyzoides, and Rothmannia wittii). Compound $\mathbf{2 3}$ was isolated from the cultured mycelium of the luminescent mushroom Neonothopanus nambi. 
1 Table 1 Compounds used in this study.

\begin{tabular}{|c|c|c|c|c|}
\hline No.* & Code & Compound & Source & References \\
\hline 1 & RL001 & $N$-methylcycloatalaphylline A & Roots of A. monophylla & (Pailee et al., 2020) \\
\hline 2 & RL006 & yukocitrine & Roots of A. monophylla & (Pailee et al., 2020) \\
\hline 3 & RL002 & $\mathrm{N}$-methylatalaphylline & Roots of A. monophylla & (Pailee et al., 2020) \\
\hline 4 & RL007 & atalaphylline & Roots of A. monophylla & (Pailee et al., 2020) \\
\hline 5 & RL004 & atalaphylline-3,5-dimethyl ether & Roots of A. monophylla & (Pailee et al., 2020) \\
\hline 6 & RL003 & 2,2-dimethylchromenocoumarin & Roots of A. monophylla & (Pailee et al., 2020) \\
\hline 7 & RL005 & auraptene & Roots of A. monophylla & (Pailee et al., 2020) \\
\hline 8 & RL009 & rubiadin-1-methyl ether & Roots of $P$. filamentosa & (Wisetsai et al., 2021) \\
\hline 9 & RL010 & rubiadin & Roots of $P$. filamentosa & (Wisetsai et al., 2021) \\
\hline 10 & RL011 & knoxiadin & Roots of $P$. filamentosa & (Wang et al., 1985) \\
\hline 11 & RL008 & nordamnacanthal & Roots of $P$. filamentosa & (Wisetsai et al., 2021) \\
\hline 12 & RL012 & damnacanthal & Roots of $P$. filamentosa & (Wisetsai et al., 2021) \\
\hline 13 & RL013 & damnacanthol & Roots of $P$. filamentosa & (Wisetsai et al., 2021) \\
\hline 14 & RL014 & 3',4',7-tri- $O$-methylluteolin & Flowers of $A$. conyzoides & (Ahond et al., 1990) \\
\hline 15 & RL015 & 4',7-di-O-methylapigenin & Flowers of $A$. conyzoides & (Ahond et al., 1990) \\
\hline 16 & RL016 & 4'-O-methylapigenin & Flowers of $A$. conyzoides & (Yim et al., 2003) \\
\hline 17 & \multicolumn{2}{|c|}{ RL017 2'-hydroxy-4,4',6'-trimethoxychalcone } & Flowers of $A$. conyzoides & (Sukari M. A, 2004) \\
\hline 18 & RL020 & 3,5-dihydroxycinnamate & Roots of $R$. wittii & (Wisetsai et al., 2020) \\
\hline 19 & RL021 & lippianoside B & Roots of $R$. wittii & (Wisetsai et al., 2020) \\
\hline 20 & RL022 & rothmannioside $\mathrm{C}$ & Roots of $R$. wittii & (Wisetsai et al., 2020) \\
\hline 21 & RL023 & rothmannioside A & Roots of $R$. wittii & (Wisetsai et al., 2020) \\
\hline 22 & RL024 & rothmannioside B & Roots of $R$. wittii & (Wisetsai et al., 2020) \\
\hline 23 & RL019 & aurisin $\mathrm{A}$ & Cultured mycelium of $N$. nam & (Kanokmedhakul et al., 2012) \\
\hline
\end{tabular}

2 Note: Compounds 1-22 were isolated from four medicinal plants (Atalantia monophylla,

3 Prismatomeris filamentosa, Ageratum conyzoides, and Rothmannia wittii). Compound 23 was

4 isolated from the cultured mycelium of the luminescent mushroom Neonothopanus nambi. 


\section{Table 2 (on next page)}

Antimycobacterial activity screening of five plant secondary metabolites against $30 \mathrm{M}$. abscessus isolates.

Note: MIC, minimum inhibitory concentration; DST, drug susceptibility testing; Acquired, acquired resistance; Inducible, inducible resistance 
1 Table 2 Antimycobacterial activity screening of five plant secondary metabolites against 30 M. abscessus isolates.

\begin{tabular}{|c|c|c|c|c|c|c|c|c|c|c|c|c|c|c|}
\hline \multirow[t]{3}{*}{ Isolates } & \multirow[t]{3}{*}{ Organism } & \multirow[t]{3}{*}{$\begin{array}{c}\text { Colony } \\
\text { morphology }\end{array}$} & \multicolumn{7}{|c|}{ MIC value of clarithromycin $(\mu \mathrm{g} / \mathrm{mL})$} & \multirow{2}{*}{\multicolumn{5}{|c|}{$\begin{array}{c}\begin{array}{c}\text { Antimycobacterial screening against } \\
\text { M. abscessus isolates }\end{array} \\
\text { MIC value of pure compounds }(\mu \mathrm{g} / \mathrm{mL})\end{array}$}} \\
\hline & & & \multicolumn{2}{|c|}{ Day 3} & \multicolumn{2}{|r|}{ Day 5} & \multicolumn{2}{|c|}{ Day 14} & \multirow[t]{2}{*}{ DST interpretation } & & & & & \\
\hline & & & MIC & Phenotype & MIC & Phenotype & MIC & Phenotype & & RL008 & RL009 & RL011 & RL012 & RL013 \\
\hline 80097 & M. abscessus subsp. abscessus & Mixed & 0.25 & Susceptible & 8 & Resistant & 16 & Resistant & Inducible & 128 & 16 & 64 & 64 & 128 \\
\hline 80167 & M. abscessus subsp. massiliense & Rough & $\geq 16$ & Resistant & $\geq 16$ & Resistant & $\geq 16$ & Resistant & Acquired & 128 & 8 & 64 & 64 & 128 \\
\hline 80225 & M. abscessus subsp. abscessus & Rough & 0.25 & Susceptible & 4 & Intermediate & $\geq 16$ & Resistant & Inducible & $<1$ & $<1$ & $<1$ & $<1$ & $<1$ \\
\hline 80448 & M. abscessus subsp. massiliense & Rough & 0.12 & Susceptible & 0.25 & Susceptible & 2 & Susceptible & Susceptible & 128 & 128 & 128 & 128 & 128 \\
\hline 80524 & M. abscessus subsp. massiliense & Mixed & 0.5 & Susceptible & 2 & Susceptible & 2 & Susceptible & Susceptible & 128 & 128 & 128 & 128 & 128 \\
\hline 80700 & M. abscessus subsp. massiliense & Rough & $>16$ & Resistant & $>16$ & Resistant & $>16$ & Resistant & Acquired & $>128$ & 128 & 64 & $>128$ & 128 \\
\hline 80824 & M. abscessus subsp. massiliense & Mixed & 0.25 & Susceptible & 0.5 & Susceptible & 1 & Susceptible & Susceptible & 128 & 16 & 32 & 128 & 128 \\
\hline 80838 & M. abscessus subsp. abscessus & Mixed & 1 & Susceptible & 16 & Resistant & 16 & Resistant & Inducible & 128 & 128 & 128 & 128 & $>128$ \\
\hline 80866 & M. abscessus subsp. massiliense & Smooth & 0.12 & Susceptible & 0.12 & Susceptible & $>16$ & Resistant & Inducible & 128 & 128 & 128 & 128 & 128 \\
\hline 80901 & M. abscessus subsp. abscessus & Rough & 1 & Susceptible & 8 & Resistant & 16 & Resistant & Inducible & $>128$ & 128 & 128 & 128 & $>128$ \\
\hline 80988 & M. abscessus subsp. abscessus & Rough & 0.12 & Susceptible & 0.25 & Susceptible & 16 & Resistant & Inducible & 128 & $>128$ & 64 & 128 & $>128$ \\
\hline 81103 & M. abscessus subsp. abscessus & Smooth & 2 & Susceptible & 2 & Susceptible & 16 & Resistant & Inducible & 128 & 128 & 64 & 128 & 128 \\
\hline 81350 & M. abscessus subsp. massiliense & Rough & $\leq 0.06$ & Susceptible & 0.12 & Susceptible & 0.12 & Susceptible & Susceptible & $>128$ & 128 & 128 & 128 & 128 \\
\hline 81422 & M. abscessus subsp. abscessus & Rough & 0.5 & Susceptible & 1 & Susceptible & $\geq 16$ & Resistant & Inducible & $>128$ & 128 & 128 & 128 & $>128$ \\
\hline 81463 & M. abscessus subsp. massiliense & Smooth & $\leq 0.06$ & Susceptible & 0.12 & Susceptible & 0.25 & Susceptible & Susceptible & 128 & 128 & 64 & 128 & 128 \\
\hline 81499 & M. abscessus subsp. massiliense & Rough & 16 & Resistant & 16 & Resistant & 16 & Resistant & Acquired & 128 & 128 & 64 & 128 & 128 \\
\hline 81618 & M. abscessus subsp. abscessus & Mixed & 16 & Resistant & 16 & Resistant & 16 & Resistant & Acquired & 128 & 16 & 32 & 128 & 128 \\
\hline 81652 & M. abscessus subsp. massiliense & Mixed & 0.12 & Susceptible & 0.25 & Susceptible & 1 & Susceptible & Susceptible & 128 & 8 & 128 & 64 & 128 \\
\hline 81702 & M. abscessus subsp. massiliense & Rough & $>16$ & Resistant & $>16$ & Resistant & $>16$ & Resistant & Acquired & $>128$ & 128 & 64 & $>128$ & 128 \\
\hline 81838 & M. abscessus subsp. massiliense & Rough & 16 & Resistant & 16 & Resistant & $\geq 16$ & Resistant & Acquired & 128 & 128 & 128 & 128 & 128 \\
\hline 82119 & M. abscessus subsp. abscessus & Smooth & 2 & Susceptible & 16 & Resistant & 16 & Resistant & Inducible & 128 & 16 & 64 & 64 & 128 \\
\hline 82154 & M. abscessus subsp. abscessus & Smooth & 0.5 & Susceptible & 8 & Resistant & 16 & Resistant & Inducible & 128 & 32 & 64 & 64 & 128 \\
\hline 82593 & M. abscessus subsp. massiliense & Rough & $\leq 0.06$ & Susceptible & 0.12 & Susceptible & 0.5 & Susceptible & Susceptible & $>128$ & 128 & 128 & $>128$ & $>128$ \\
\hline
\end{tabular}




\begin{tabular}{|c|c|c|c|c|c|c|c|c|c|c|c|c|c|c|}
\hline 82895 & M. abscessus subsp. massiliense & Rough & 0.25 & Susceptible & 0.5 & Susceptible & 2 & Susceptible & Susceptible & 128 & $>128$ & 32 & 128 & $>128$ \\
\hline 82998 & M. abscessus subsp. abscessus & Smooth & 16 & Resistant & 16 & Resistant & 16 & Resistant & Acquired & 128 & 128 & 128 & 128 & 128 \\
\hline 83305 & M. abscessus subsp. abscessus & Smooth & 16 & Resistant & 16 & Resistant & 16 & Resistant & Acquired & $>128$ & 128 & 128 & 128 & 128 \\
\hline 83310 & M. abscessus subsp. abscessus & Rough & 8 & Resistant & 8 & Resistant & $\geq 16$ & Resistant & Acquired & 128 & $>128$ & 128 & $>128$ & $>128$ \\
\hline 83347 & M. abscessus subsp. massiliense & Mixed & 0.25 & Susceptible & 0.5 & Susceptible & 0.5 & Susceptible & Susceptible & 128 & 128 & 128 & 128 & $>128$ \\
\hline 90919 & M. abscessus subsp. massiliense & Mixed & 0.12 & Susceptible & 0.12 & Susceptible & 0.5 & Susceptible & Susceptible & 128 & 128 & 128 & 128 & $>128$ \\
\hline 826492 & M. abscessus subsp. massiliense & Mixed & 0.12 & Susceptible & 0.25 & Susceptible & 16 & Resistant & Inducible & 128 & 16 & 64 & 64 & 128 \\
\hline
\end{tabular}

2 Note: MIC, minimum inhibitory concentration; DST, drug susceptibility testing; Acquired, acquired resistance; Inducible, inducible

\section{3 resistance}




\section{Table 3 (on next page)}

Synergistic activity of five plant secondary metabolites combined with clarithromycin against 14 clarithromycin-resistant $M$. abscessus isolates.

Note: MIC, minimum inhibitory concentration; CLA, clarithromycin; Acquired, acquired resistance; Inducible, inducible resistance; $\mathrm{FICl}$, fractional inhibitory concentration index $\mathrm{FICl}$ interpretation: $\leq 0.5$ : synergy; $>0.5-1.0$ : additive; $>1.0-4.0$ : indifference; $>4.0$ : antagonism (Doern 2014) Grey-shaded boxes shows synergistic effect 
1 Table 3 Synergistic activity of five plant secondary metabolites combined with clarithromycin against 14 clarithromycin-resistant $M$.

2 abscessus isolates.

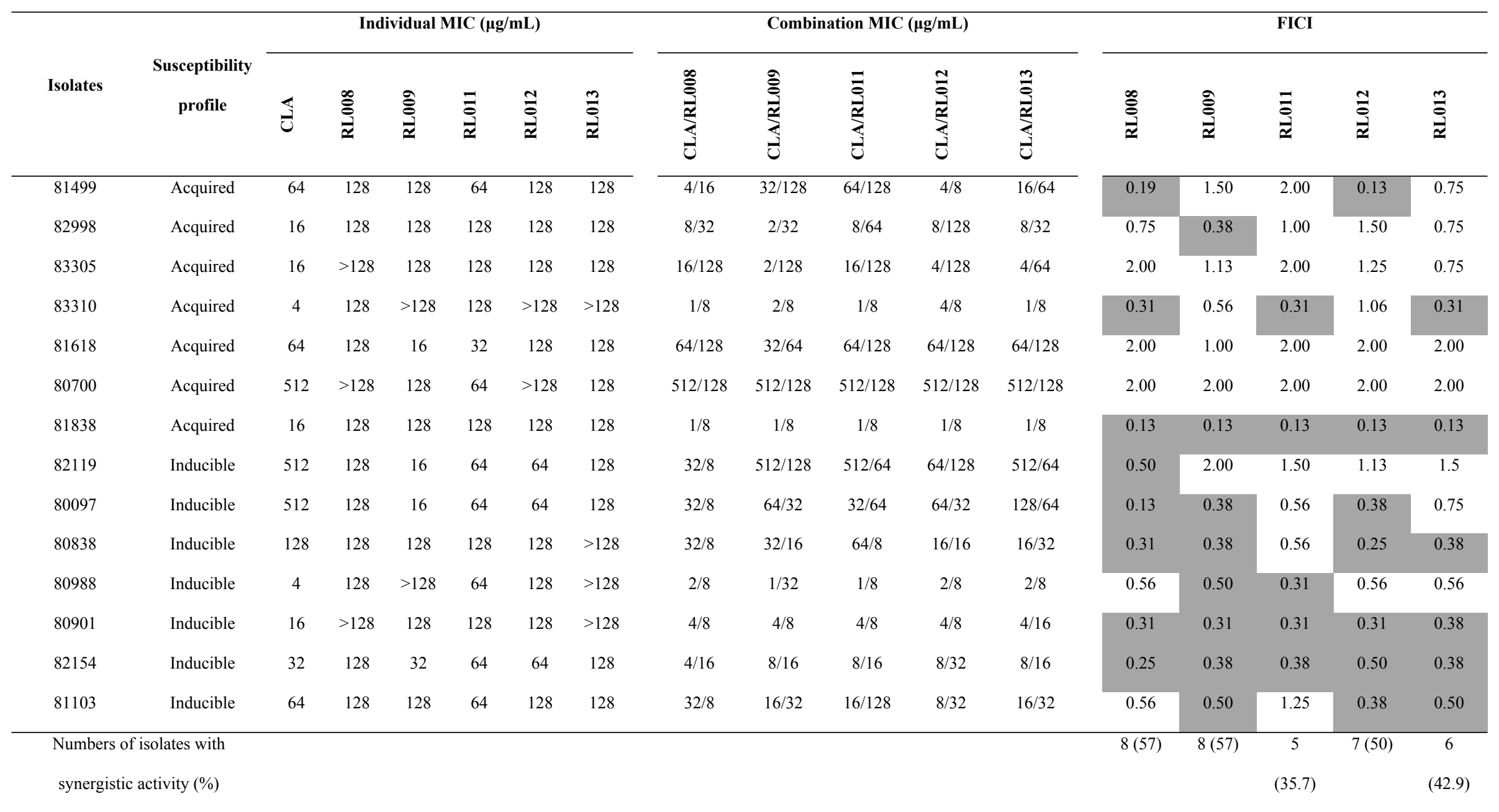


3 Note: MIC, minimum inhibitory concentration; CLA, clarithromycin; Acquired, acquired resistance; Inducible, inducible resistance;

$4 \quad$ FICI, fractional inhibitory concentration index

$5 \quad$ FICI interpretation: $\leq 0.5$ : synergy; $>0.5-1.0$ : additive; $>1.0-4.0$ : indifference; $>4.0$ : antagonism (Doern 2014)

6 Grey-shaded boxes shows synergistic effect 


\section{Figure 1}

Characteristics of 30 Mycobacterium abscessus isolates and antimycobacterial activities of five plant secondary metabolites.

All 30 isolates were either M. abscessus subspecies abscessus (red box) or subspecies massiliense (red border) based on genome analysis. A bootstrap consensus tree was inferred from 1,000 replicates. The phylogenetic tree was visualized using iTol software (https://itol.embl.de/). Red circles refer to bootstrap values and the size of each circle is proportional to its value (the largest red circle indicates a value of 100\%). Mycobacterium chelonae was used as the outgroup and three reference strains of $M$. abscessus were included for comparison. Colony morphology was classified as rough (blue box), smooth (light green box) or mixed (purple box). Clarithromycin (CLA) susceptibility profiles showed acquired resistance (red box), inducible resistance (orange box), or susceptibility to CLA (green box). MIC values for the five compounds tested against the M. abscessus isolates ranged from 8-128 $\mu \mathrm{g} / \mathrm{mL}$ (open circles) or were $>128 \mu \mathrm{g} / \mathrm{mL}$ (shaded circles). Light blue, blue, dark blue, dark purple and purple circles refer to compounds labeled as RL008, RL009, RL011, RL012, and RL013, respectively. Synergistic activity of compounds in combination with clarithromycin is represented as no synergism (open box) and synergism (shaded box). Pink, red, dark blue, yellow and light green boxes refer to combinations RL008/CLA, RL009/CLA, RL011/CLA, RL012/CLA, and RL013/CLA, respectively. 


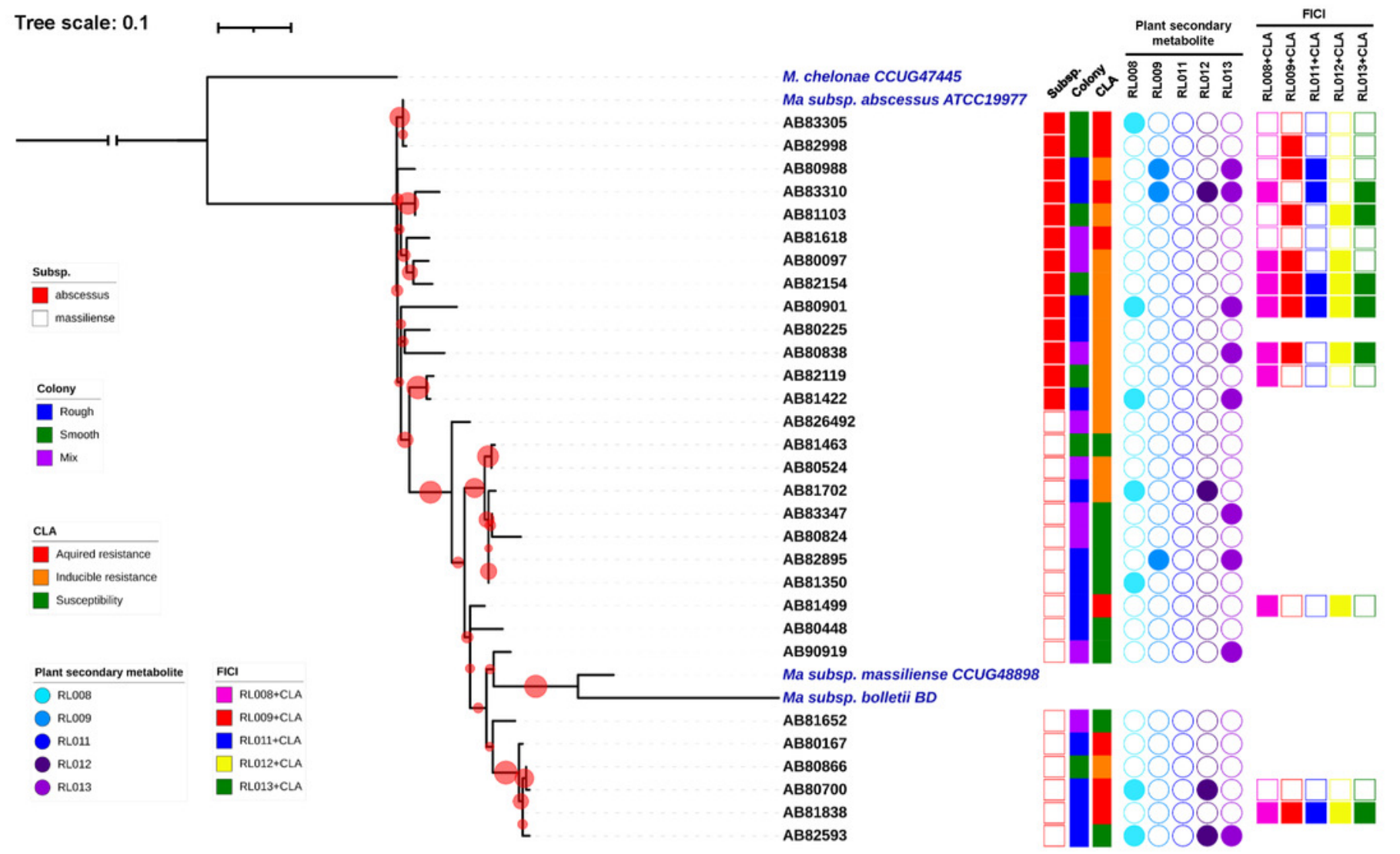


Figure 2

Hemolytic activity of plant secondary metabolites against red blood cells (RBCs).

RBC suspension was incubated for $1 \mathrm{~h}$ with different concentrations of (A) RL008, (B) RL009, (C) RL011, (D) RL012 and (E) RL013. The hemolytic activity is presented as the percentage of hemolysis. Data are expressed as mean. Note: When the \%hemolysis of the tested concentration is lower than the control (negative value), $0 \%$ hemolysis is used as the baseline. Concentrations of secondary metabolites before adding RBC suspension are shown.
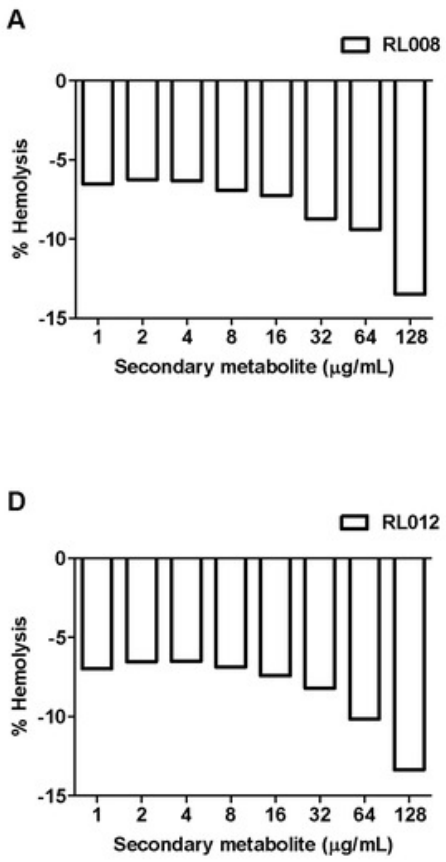

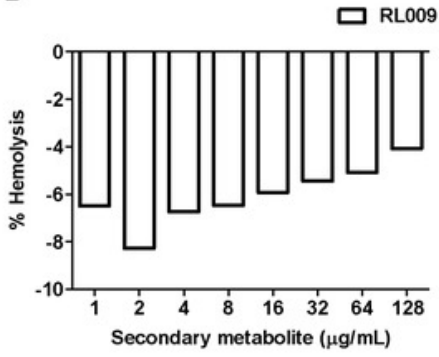

E

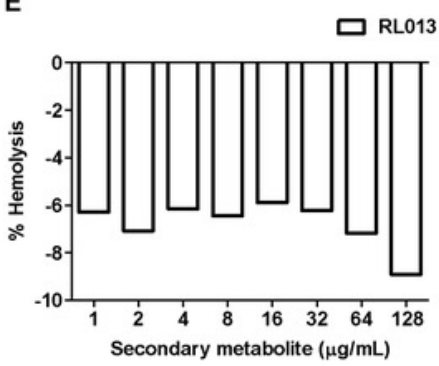

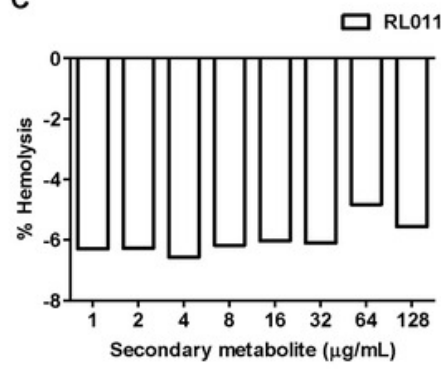

$\mathbf{F}$

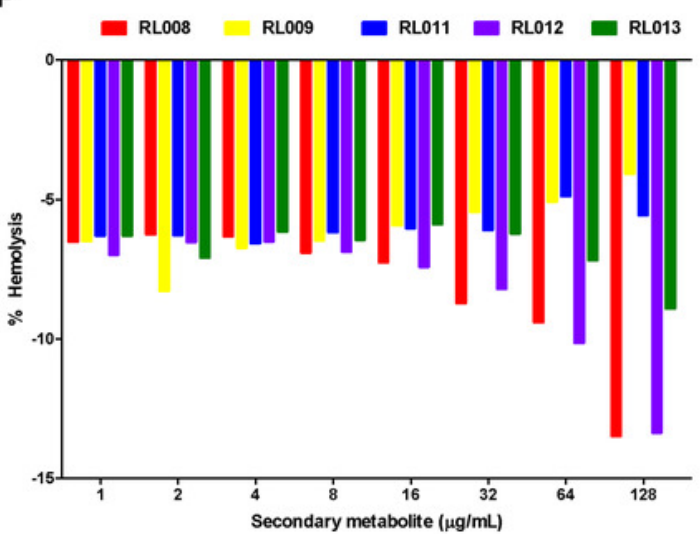




\section{Figure 3}

Red blood cell (RBC) morphology under the light microscope.

RBC suspension was incubated for $1 \mathrm{~h}$ with (A) $1 \%$ Triton-X (positive control, showing complete hemolysis), (B) 1\% PBS (negative control, showing no hemolysis) and (C) 128 $\mu \mathrm{g} / \mathrm{mL}$ of RL013 (showing some shrinkage due to osmotic effects). With the remaining four compounds, RBCs exhibited similar morphology of cell shrinkage, especially at high MICs.
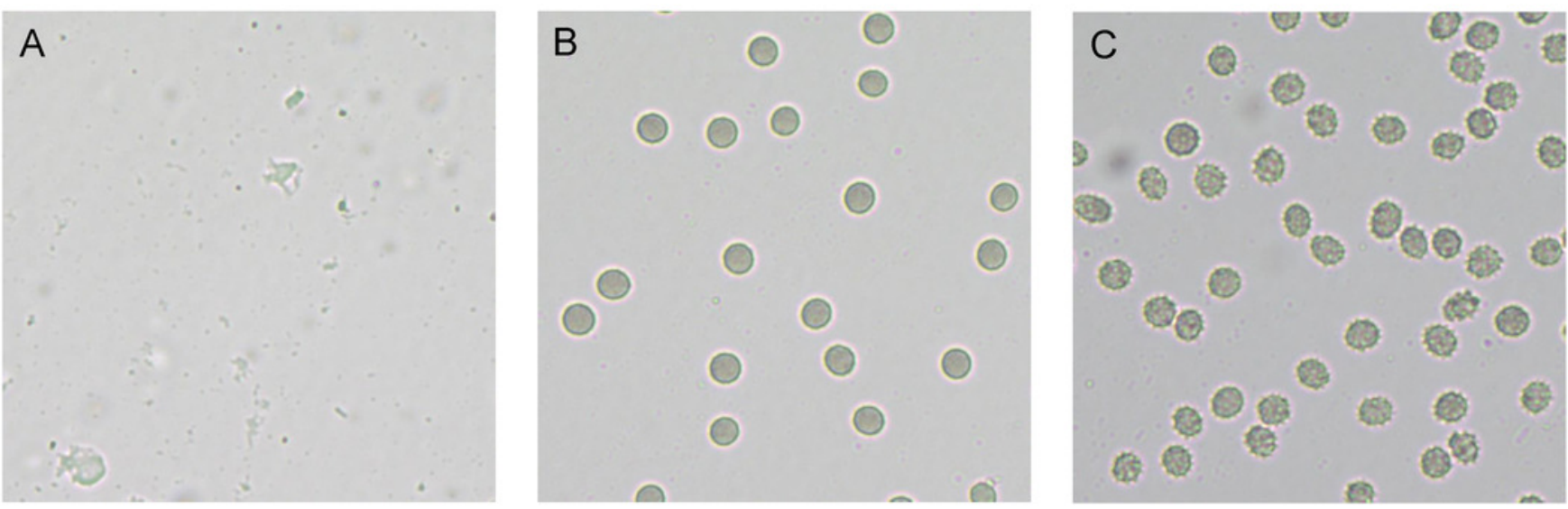
Figure 4

Effect of plant secondary metabolites on white blood cells (WBCs).

WBCs were incubated for $1 \mathrm{~h}$ with different concentrations of (A) RL008, (B) RL009, (C)

RL011, (D) RL012, (E) RL013 and (F) One-way ANOVA followed by post-hoc LSD test was used to determine significant differences $\left({ }^{*} P<0.05\right)$. The toxicity of each secondary metabolite is expressed as the percentage of dead cells. Data are expressed as means.
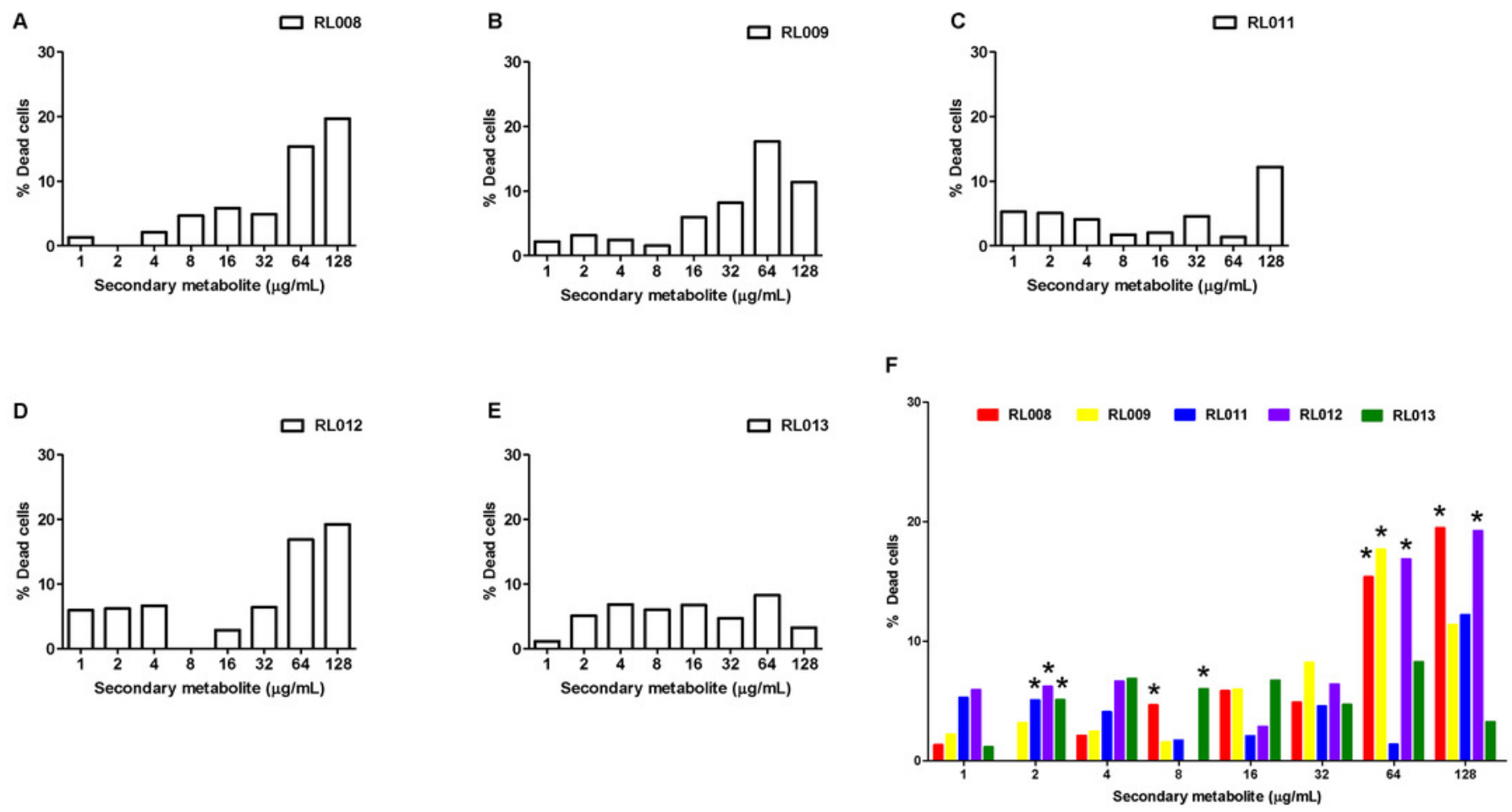